\title{
Translational medicine should translate medical science and technology into health care for everyone in China
}

\author{
CHEN Zhu ${ }^{1,2 *} \&$ ZHOU GuangBiao ${ }^{3}$ \\ ${ }^{1}$ State Key Laboratory of Medical Genomics and Shanghai Institute of Hematology, Ruijin Hospital, Affiliated to Shanghai Jiao Tong Univer- \\ sity School of Medicine, Shanghai 200025, China; \\ ${ }^{2}$ Ministry of Health, Beijing 100044, China; \\ ${ }^{3}$ Division of Molecular Carcinogenesis and Targeted Therapy for Cancer, State Key Laboratory of Biomembrane and Membrane Biotechnol- \\ ogy, Institute of Zoology, Chinese Academy of Sciences, Beijing 100101, China
}

Received September 15, 2011; accepted September 29, 2011

Citation: Chen Z, Zhou G B. Translational medicine should translate medical science and technology into health care for everyone in China. Sci China Life Sci, 2011, 54: 1074-1076, doi: 10.1007/s11427-011-4231-5

Over the years, a chasm between biomedical researchers and the patients who may benefit from their discoveries has been opened. On one hand, millions of patients with diseases such as cancer are anxiously waiting for new remedies to save their lives. On the other hand, many exciting basic science discoveries do not have opportunities to find practical applications. Recently emerging translational medicine aims to tie basic research to clinical results and optimize both patient care and preventive measures.

\section{Translational medicine is a people-oriented medical practice}

Translational medicine converts promising laboratory discoveries into clinical applications and elucidates clinical questions with the use of benchwork, aiming to facilitate prediction, prevention, diagnosis, and treatment of diseases. It is regarded as a two-way road-bench to bedside and bedside to bench - and requires close collaboration between clinicians/public health professionals and basic scientists. With the ultimate goal to develop more effective preventive/therapeutic approaches and improve clinical outcomes and health levels, translational medicine is therefore a peo-

*Corresponding author (email: zchen@stn.sh.cn) ple (patients and the general population as a whole)-oriented medical practice.

\section{Challenges for China's health care system}

The past three decades have witnessed tremendous advances in China in the development of living conditions, food and nutrition, and the health care system. However, while the economy grows and society rapidly transforms, the health care system faces multiple problems (Table 1). For example, rapid industrialization and urbanization may perturb ecology, pollute the environment, and even affect residents' homes. In some regions, occupational, food, and drug safety should be strengthened and sanitary drinking water and toilet systems should be provided to the residents.

China bears a complex disease spectrum (Table 2). On one hand, communicable diseases frequently seen in developing countries remain a heavy burden. For example, China has approximately 100 million and 4.5 million people infected with the hepatitis B virus and tuberculosis, respectively. Each year there are 800000 new cases of traditional sexually transmitted diseases, and there are an estimated 700000 people living with human immunodeficiency virus. On the other hand, chronic diseases commonly found in developed countries are also the leading causes of death and 
Table 1 Social transformation and related health problems in China

\begin{tabular}{ll}
\hline Social transformation & Consequence and major health problems \\
\hline Rapid industrialization & Ecological degradation; environmental pollution; toxins; occupational hazards \\
Rapid progress of market economy & Problems in employment and social security; lifestyle and stress \\
Rapid urbanization & Problems in living conditions, housing, transportation; stress and social security \\
Rapid globalization & Cross-border disease transmission \\
Aging population & Chronic diseases; health care and social security for aged people \\
Change of lifestyle & Disease and death associated with unhealthy lifestyle \\
\hline
\end{tabular}

Table 2 Major diseases in China

\begin{tabular}{|c|c|c|c|}
\hline \multicolumn{2}{|l|}{ Major communicable disease } & \multicolumn{2}{|l|}{ Major chronic disease } \\
\hline Infectious disease & Accounts for $12 \%$ of deaths & Chronic disease & $\begin{array}{l}\text { Leads to shortening of life span by } \\
49 \% \text {. }\end{array}$ \\
\hline Hepatitis B & $\begin{array}{l}\text { More than } 100 \text { million people are infected with } \\
\text { the hepatitis B virus, and } 300000 \text { people die of } \\
\text { hepatitis B virus-related diseases every year. }\end{array}$ & Cancer & $\begin{array}{l}\text { Cancer accounts for some } 1890000 \\
\text { deaths each year. }\end{array}$ \\
\hline Tuberculosis & $\begin{array}{l}\text { China has } 4.5 \text { million tuberculosis cases annually; } \\
160000 \text { people succumbed to this disease in } \\
2008 \text {. }\end{array}$ & Heart disease & $\begin{array}{l}\text { Each year approximately } \\
1250000 \text { people die of heart disease. }\end{array}$ \\
\hline Traditional sex transmitted disease & Each year there are 800000 new cases in China. & Stroke & $\begin{array}{l}\text { Cerebral stroke causes } 1900000 \\
\text { deaths each year. }\end{array}$ \\
\hline $\begin{array}{l}\text { Acquired immunodeficiency syn- } \\
\text { drome (AIDS) }\end{array}$ & More than 700000 cases living with HIV/AIDS & Hypertension & $\begin{array}{l}\text { There are } 200 \text { million patients with } \\
\text { hypertension in China. }\end{array}$ \\
\hline Emerging infectious diseases & Swine flu (H1N1), avian flu & Diabetes mellitus & $\begin{array}{l}\text { There are } 90 \text { million patients with } \\
\text { diabetes in China. }\end{array}$ \\
\hline
\end{tabular}

disability in China. For example, cancer and cardiovascular disease represent the two leading causes of death, and hypertension and diabetes mellitus are diagnosed in some 200 million and 90 million people annually in China, respectively. These data demonstrate that the health care system in China is facing great challenges, and a state effort is needed to meet these challenges.

\section{Principles for development of translational medicine in China}

China is deepening its reform to improve its people's welfare. The development of translational medicine will accelerate disease control and finding solutions for health problems, with the following principles being emphasized.

\subsection{Close collaboration}

Translational medicine is a multidisciplinary program that integrates research from the medical sciences, basic sciences, and social sciences, with the aim of optimizing patient care and preventive measures that may extend beyond health care services. Pharmaceutical or biotech companies that use a patient-driven approach to drug development also play an important role in translational medicine. Therefore, close collaboration among clinical practitioners, basic researchers, biotechnologists, ethicists, sociologists, and others is essential to the development of translational medicine. Moreover, translational medicine is a medical practice based on interventional epidemiology. Policy-makers and those in government may facilitate the practice not only by funding support, but also by organizing social resources and evidence-based policy-making and transforming discoveries in translational research into public health services and preventive approaches.

\subsection{Etiology and prevention of disease}

Environmental factors are responsible for diseases from infections to immunologic diseases and cancer. Therefore, translational research should identify specific environmental factors that cause health problems, develop methods to eliminate these factors, and investigate preventive strategies to decrease potential harm to residents. Furthermore, pathogenesis of environmental health problems should be elucidated using genomic, proteomic, and other systematic technologies. These approaches are important, especially for regional disease caused by environmental factors. These efforts may facilitate science- and evidence-based policy-making and will in turn greatly reduce disease burden and improve people's welfare.

\subsection{Identification of biomarkers for early diagnosis}

Identification of biomarkers for early diagnosis of diseases such as cancer remains an urgent need to improve the clinical outcome of patients. Advances in genomics, proteomics, epigenomics, and molecular pathology will provide many candidate biomarkers with potential clinical value. 


\subsection{Translating science and technology into grass- roots health care}

China remains a developing country whose disease burden is huge, while a large proportion of its residents are unable to afford their prescription drugs because of abject poverty. The level of reimbursement from medical insurance is very limited, especially for diseases such as cancer, although more than 1.27 billion people have been covered by rural and urban medical insurance to date. Hence, reliable and cost-effective biotechnologies/bioremediations should be encouraged in translational research. A paradigm for this is the treatment of acute promyelocytic leukemia, which was considered to be the most fatal type of leukemia five decades ago. The application of all-trans retinoic acid and arsenic trioxide attains a high complete remission rate, while combination of the two plus chemotherapy achieves a five-year overall survival of $>90 \%$ for all patients, making acute promyelocytic leukemia a curable disease. Interestingly, according to the statistics from the Shanghai Institute of Hematology, Ruijin Hospital, the overall cost of using this combinatory regimen is generally within CNY (RMB) 100000 , while allogeneic transplantation, which only obtains a five-year survival rate of $60 \%-70 \%$, costs CNY 300000-400000. Another example comes from Shanghai Children's Medical Center in treating acute lymphoid leukemia, which accounts for $80 \%$ of childhood leukemia. This center establishes a standard childhood acute lymphoid leukemia treatment protocol in which patients receive prednisone, vincristine, daunorubicin, cyclophosphamide, cy- tarabine, dexamethasone, and L-asparaginase according to their risk stratification. Intriguingly, overall continuous complete remission reaches $80 \%$, while total costs per patient are under CNY 80000. At present, quality treatment services for these two categories of leukemia among children in all rural areas of China are covered by the New Rural Cooperative Medicare Scheme, with a reimbursement rate of $90 \%$. These two examples demonstrate that innovation and cost-effectiveness could be fulfilled and that the best practices in clinical study should be transformed into policy-making in translational medicine.

\section{Concluding remarks}

The Chinese government strives to protect and promote citizens' health and improve their welfare. In 2009, China launched long-awaited health reforms with an investment plan of CNY 850 billion (USD 124 billion) for the first three years (2009-2011) to improve the medical and health care system to achieve universal access to basic medical and health services for all of its citizens. Moreover, the 12th Five-year Socio-economic Development Plan (2011-2015) has been launched, which includes as part of the core indicator health-related objectives such as the increase of one year of life expectancy. Translational medicine, a multidisciplinary practice, should help to cultivate health system innovation and health industry growth and facilitate the construction of a harmonious society.

Open Access This article is distributed under the terms of the Creative Commons Attribution License which permits any use, distribution, and reproduction in any medium, provided the original author(s) and source are credited. 